\title{
Multi-Functional Device for Cardiologic Telemedicine and Diagnostic Holter
}

\author{
A Belardinelli, L Muratori, I Corazza, M Magnalardo, \\ F Marangoni, R Zannoli \\ Cardiovascular Department, University of Bologna, Bologna, Italy
}

\begin{abstract}
Although different systems are available on the market to record ECG and to send it to an analisys station, there is still demand for a more vesatile, easy to use device that shorten the time the diagnosis is available, avoiding the patient to move to a healthcare center: the purpose of our work was to validate a solution that fullfil this requirement. Twenty family doctors and two cardiologists were involved in the evaluation of the whole solution and 400 exams were performed. The results showed that the device is well tolerated by patients and the workflow allures the practitioners for the combination of its semplicity and effectiveness in reducing the time to reach the diagnosis, although a remarkable number of cardiac traces were not good enough to formulate a diagnosis, mainly due to contact loss in the patient's electrical circuit. An investigation was carried on to identify the causes of bad electrical connections and corrective measures were taken to improve the number of good quality signals.
\end{abstract}

\section{Introduction}

A major requirement for cardiologist is to prevent severe injuries by monitoring the patients not only during the hospitalization but also when the patient is at home, followed by the family doctor. Moreover, the possibility to perform standard ECG at patient's home or at family doctor ambulatory is mandatory to reduce the costs of hospitalizations and cardiologic rehabilitation procedures [1-7]. Nowadays, different systems are available to record ECG and to send it to the hospital via phone or internet connection, where a specialist can read the data and decide the best strategy to improve the therapy. [811] These devices can present different levels of complexity, depending on their clinical aim: from the standard ECG recorder able to acquire 12 signals to the simplified system to acquire up to 3 channels at a low resolution but for a longer time to evaluate arrhythmias. Both these approaches present some problems connected or to the difficulty for the patient to manage the acquisition and then the transmission to the hospital or to the high specialization and the low versatility of the devices [12-13].

The purpose of our work was to validate a versatile solution that comprises a powerful and easy to use instrument (TH16, Q\&S, Reggio Emilia, Italy) and an internet application (QS-32 NET) verifying the feasibility of: (a) performing standard ECG or 24 hours holter recordings in a general practice contest; (b) sending data to a telemedicine centre to be stored and completely managed by an user-friendly software; (c) making the specialist able to connect to the server from a remote client, analyse ECG recordings and make the diagnosis; (d) allowing the family doctor to connect to the server from a remote client as well and consult the specialist's report.

\section{Methods}

TH16 was designed for family doctors, making them able to perform standard ECG, holter recordings during pathologic events and then to transmit the data to the server station via phone line. By simply programming the device, TH16 can be configured to acquire and store up to 20 standard ECG recordings or a 3 channels 24 hours holter monitoring. Firmware on TH16 allows self background analysis to identify and mark suspicious events to help the doctor in the diagnosis. After acquisition, data are sent to the telemedicine centre using the integrated modem. The software on the server automatically links the data to the patients archive, allows the specialist to view the recordings and make the diagnosis, allows the family doctor to view the specialist's report to adapt the therapy of the patient. The system (fig. 1) is set up by a central server with a dedicated software (QS-32 NET) to store and manage ECG data. Doctors and specialists can access the system through a simple internet connection to insert new patients and analyse the ECG recordings. Communication between the TH16 and the server is digital and is performed via either a PSTN or a GSM modem, according to the particular assembly of the device. 


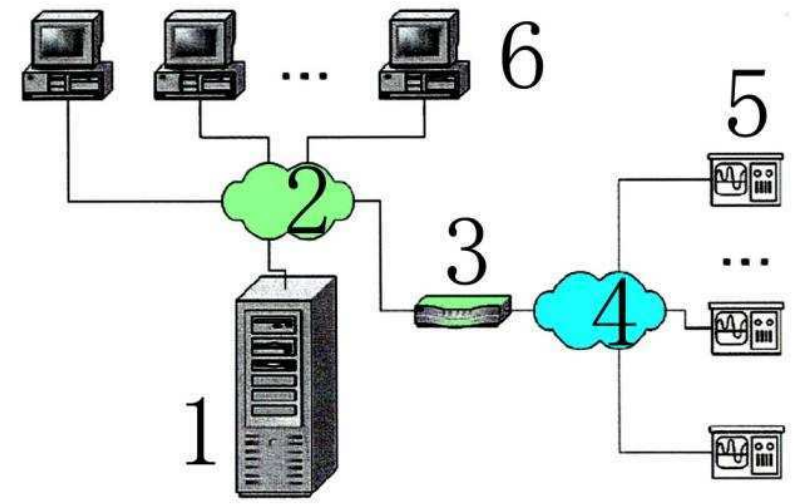

Figure 1: System architecture. (1) Server; (2) Internet connection; (3) Router; (4) Phone Line; (5) TH16, (6) Clients.

Although it is a light handheld device, TH16 is equipped with a six keys keyboard and a 240x320 display, which make the device capable of performing sophisticated user interface features, such as checking the correctness of the electrode positions before starting the acquisition, reviewing the acquired data and managing the internal ECGs archive, allowing the patient to insert notes and the simpthons that he/ her feels during the holter monitoring, driving the patient or the doctor during the trasmission of the ECGs to the server.

TH16 is equipped with a modem supporting a software interface capable of performing PPP connection and the main internet protocols, such as DNS and FTP, which allows the device to send the central server the recorded ECGs. The transferred data are kept in binary format and encrypted with a proprietary algorithm for safety reasons. The application software assures the integrity and the completeness of the trasmission, allowing a self retry functionality in case of unexpected errors and providing the user with trasmission report on the display.

On the other hand, data exchange between the central server and the family doctor or the specialists is carried on via internet through an SSL connection, which ensure data encription and integrity. The job of doctors for evaluating the ECG and making the reports is done in an easy way due to the user friendly web interface. The web server application connects to an Oracle database and is designed to support the archiving of additional patient data, including any kind of document format.

TH16 testing involved 2 cardiologists and 20 family doctors previously skilled about the functioning and the employment of TH16. 174 ECG and 230 holter recordings were performed in the ambulatory or at the patient home and transmitted to the telemedicine server. Each patients gave the written consent to participate at the study and no selection on the basis of the subjects clinical conditions was made.
The correctness of the transmission was evaluated by comparing the original signal with the received one; then both the specialists analyzed the recordings to evaluate the quality of the signals and to make the diagnosis.

\section{Results}

An example of good transmitted signal is shown in figure 2 .

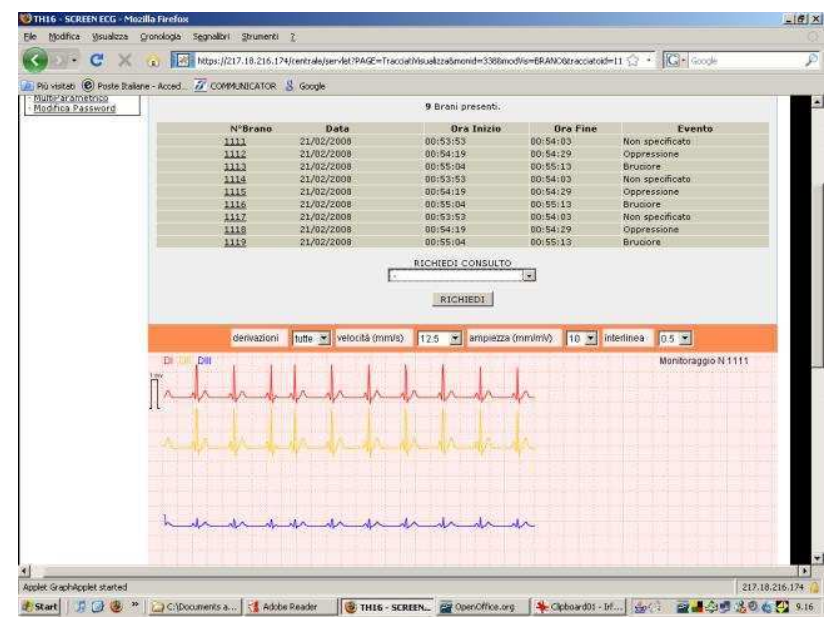

Figure 2: example of ECG signals tansmitted from TH16 to the server.

All the recordings were correctly transmitted and received from the server. In $10(6+4)$ cases, transmission was repeated twice because of lack in phone line connection. Nevertheless, all the data were properly received from the server. The $86 \%$ of ECG recordings were classified of good quality from both the specialists and then an analysis was performed. Only the $48 \%$ of holter signals were considered readable from the 2 specialists and so only 110 recordings were analysed. The $14 \%$ of ecg and the $52 \%$ of holter signals were impossible to analyse because of the artefacts due to unstable or wrong positioning of the electrodes or the whole lack of the electrical connection between the patient skin and the device.

Results are summarized in table 1.

\begin{tabular}{|l|c|c|c|c|}
\hline \multicolumn{1}{|c|}{ Number of } & \multicolumn{2}{c|}{ ECG } & \multicolumn{2}{c|}{ Holter } \\
\hline Recorded signals & \multicolumn{2}{c|}{174} & \multicolumn{2}{c|}{230} \\
\hline Received signals & 174 & $100 \%$ & 230 & $100 \%$ \\
\hline Readable signals & 150 & $86 \%$ & 110 & $48 \%$ \\
\hline Written Reports & 150 & $86 \%$ & 110 & $48 \%$ \\
\hline
\end{tabular}

Table 1: results of the experimentation

All the diagnosis were performed within 24 hours from the transmission and the feedback to the family doctor 
was immediate.

\section{Discussion and conclusions}

TH16 demonstrated to be a powerful and easy to use device, well tolerated by patients. The intermediary role of the family doctor between patient and specialist represent the best solution in order to optimize the procedures and refine the diagnosis [2, 3, 4, 7, 13]. More improvements are necessary in order to minimize bad quality acquisitions: $48 \%$ of correct holter recordings is a very poor percentage and must be enhanced. A more accurate investigation has been performed about the way the signal quality could be improved, which finally lead to both a new design of the mechanical connection between the patient cable and the device, in order to make it more secure, and the necessity of a firmware revision to force the practitioners to use the feature of the device for immediate control of the acquired signal quality. Such corrctive measures should reduce the possibility to acquire ECG signal with artefact or completely unreadable. Moreover, for it is needed to avoid bad acquisitions due to incorrect usage of the device, including erroneuos electrode positioning, a closer collaboration between family doctors, specialists and tehnicians, beginning from the initial training, is desiderable.

\section{References}

[1] Brunetti ND, Amodio G, De Gennaro L, Dellegrottaglie G, Pellegrino PL, Di Biase M, Antonelli G. Telecardiology applied to a region-wide public emergency health-care service. J Thromb Thrombolysis 2008; in press.

[2] Daudelin DH, Selker HP. Medical error prevention in ED triage for ACS: use of cardiac care decision support and quality improvement feedback. Cardiol Clin 2005; 23: 601-614.

[3] Fahey T, Jeyaseelan S, McCowan C, Carr E, Goudie BM, Pringle SD, Donnan PT, Sullivan FM, Struthers AD. Diagnosis of left ventricular systolic dysfunction (LVSD): development and validation of a clinical prediction rule in primary care. Fam Pract 2007; 24:628-635.

[4] Fitzmaurice DA, Hobbs FDR, Jowett S, Mant J, Murray ET, Holder R, Raftery JP, Bryan S, Davies M, Lip GYH, Allan TF. Screening versus routine practice in detection of atrial fibrillation in patients aged 65 or over: cluster randomised controlled trial. BMJ 2007; 335;383.

[5] Fletcher GF, Chiaramida AJ, LeMay MR, Johnston BL, Thiel JE, Spratlin MC. Telephonically-monitored home exercise early after coronary artery bypass surgery. Chest 1984;86;198-202.

[6] Molinari G, Reboa G, Frascio M, Leoncini M, Rolandi A, Balzan C, Barsotti A. The role of telecardiology in supporting the decision-making process of general practitioners during the management of patients with suspected cardiac events. J Telemed Telecare
2002;8(2):97-101.

[7] Scalvini S, Zanelli E, Volterrani M, Castorina M, Giordano A, Glisenti F. Potential cost reductions for the National Health Service through a telecardiology service dedicated to general practice physicians. Ital Heart J 2001; 2(10):1091-7.

[8] Motoi K, Kubota S, Ikarashi A, Nogawa M, Tanaka S, Nemoto T, Yamakoshi K. Development of a fully automated network system for long-term health-care monitoring at home. Proceedings of the 29th Annual International Conference of the IEEE EMBS Cité Internationale, Lyon, France August 23-26, 2007; 18261829.

[9] Rothman SA, Laughlin JC, Seltzer J, Walia JS, Baman RI, Siouffi SJ, Sangrigoli RM, Kowey PR. The Diagnosis Of Cardiac Arrhythmias: A Prospective Multi-Center Randomized Study Comparing Mobile Cardiac Outpatient Telemetry Versus Standard Loop Event Monitoring. J Card Electrophysiol 2007; 18(3): 241-247.

[10] Scherr D, Dalal D, Henrikson CA, Spragg DD, Berger $\mathrm{RD}$, Calkins H, Cheng A. Prospective comparison of the diagnostic utility of a standard event monitor versus a "leadless" portable ECG monitor in the evaluation of patients with palpitations. J Interv Card Electrophysiol 2008; 22:39-44.

[11] Iwamoto J, Yonezawa Y, Maki H, Ogawa H, Ninomiya I, Sada K, Hamada S, Hahn AW, Caldwell WM. A mobile phone-based ECG monitoring system. Biomed Sci Instrum. 2006;42:217-22.

[12] Funk M, Chrostowski VM, Richards S, Serling SA. Feasibility of using ambulatory electrocardiographic monitors following discharge after cardiac surgery. Home Healthcare Nurse 2005; 23(7): 441-449.

[13] Jensen MSA, Thomsen JL, Jensen SE, Lauritzen T, Engberg M. Electrocardiogram interpretation in general practice. Fam. Pract 2005; 22:109-113.

Address for correspondence:

Lorenzo Muratori

Q\&S S.p.a.

Via R. Livatino, 9

42100 Reggio Emilia

ITALIA

lorenzo.muratori@quesse.it 